\title{
Projection of 5 Dimensions into Four Is When the Octonionic Structure Kicks in as an Emergent Gravity Phenomenon
}

\author{
Andrew Walcott Beckwith \\ Physics Department, College of Physics, Chongqing University, Chongqing, China \\ Email: Rwill9955b@gmail.com
}

How to cite this paper: Beckwith, A.W. (2018) Projection of 5 Dimensions into Four Is When the Octonionic Structure Kicks in as an Emergent Gravity Phenomenon. Journal of High Energy Physics, Gravitation and Cosmology, 4, 731-742. https://doi.org/10.4236/jhepgc.2018.44041

Received: April 29, 2017

Accepted: October 15, 2018

Published: October 18, 2018

Copyright $\odot 2018$ by author and Scientific Research Publishing Inc. This work is licensed under the Creative Commons Attribution International License (CC BY 4.0).

http://creativecommons.org/licenses/by/4.0/

\begin{abstract}
The quantum gravity problem that the notion of a quantum state, representing the structure of space-time at some instant, and the notion of the evolution of the state, does not get traction, since there are no real "instants", is avoided by having initial octonionic geometry embedded in a larger, nonlinear (semi-classical) embedding structure. We detail some of what the quantum HUP is, in terms of deterministic 5-dimensional geometry and show that the projection of 5 dimensions into four is when the octonionic structure kicks in as an emergent gravity phenomenon. The example of such is to consider what would happen if there was an aftermath to a presumed initial causal discontinuous structure, after math being the generation of millions of Planck mass black holes, which would in themselves generate emergent gravity.
\end{abstract}

\section{Keywords}

Octonionic Geometry, Modified HUP, Embedding of Quantum HUP in Deterministic Structure

\section{What Is Special about Octonionic Structure? Why Should One Care about It?}

Our plan is as follows. We state the Modified HUP results, as a Pre-Octonionic space-time result, and then we will specify that we are transitioning to Octonionic space time. The transition to Octonionic space time will then preserve one 
key result that we have, due to the earlier Pre-Octonionic space-time, a minimum time step.

In a word, this is the setup of the new physics, plus our resolution

1) In Pre-Octonionic (Pre-Planckian) Space-time there exist conditions for which we form an initial smallest time step, and that the Pre-Planckian Space-time is where we specify initially a modified HUP (Heisenberg Uncertainty principle).

2) In Octonionic (Planckian) Space-time, we recover QM and the usual HUP, but also, we have the benefits of keeping the minimum time step as to what is given from the Pre-Octonionic structure.

3) To get "instants" of time, we need to go beyond the standard model. After having said this, let us go to the construction of Octonionic non-commutative geometry.

4) We will conclude with how specific properties of octonion numbers algebra influence the structure and behavior of the early-cosmology model. What one of the referees reviewing this document did not realize is that the Octonionic space time closely conforms to the Standard Model of physics, as has been stated repeatedly, and that the Pre-Octonionic state is when the Standard Model of physics does not apply. i.e. the division line between the Pre-Octonionic Model and the Octonionic model directly correlates a transformation from Pre-Planckian physics to Planckian physics.

5) We discuss the transformation from the Pre-Octonionic state to the Octonionic in terms of the following equations as given below.

6) The Transition from Pre-Octonionic to octonionic gravity and causal discontinuity issues will then be presented, as an embedding of quantum structures in a 5-dimensional deterministic setting.

Keep in mind one basic fact: If we restrict ourselves solely to Octonionic geometry, we are embedded deeply in only what the Standard Model of physics allows. We should though understand what is implied by the physics of the Octonionic structure and so the rest of this first discussion is devoted to it. First of all we bring up causal discontinuity and the Friedman equation.

\section{What Leads to Casual Discontinuity in Scale Factor Evolution}

The Friedmann equation [1] for the evolution of a scale factor $a(t)$,

$$
\left(\frac{\dot{a}}{a}\right)^{2}=\frac{8 \pi G}{3} \cdot\left(\rho_{\text {rel }}+\rho_{\text {matter }}\right)+\frac{\Lambda}{3}
$$

suggests a non partially ordered set evolution of the scale factor with evolving time, thereby implying a causal discontinuity. The validity of this formalism is established by rewriting the Friedman equation as follows [2]: $a\left(t^{*}\right)<l_{P}$ for $t^{*}<t_{P}=$ Planck time, and $a_{0}=l_{P}$, for a discrete equation model of Equation (1) leads to 


$$
\begin{aligned}
& \frac{a\left(t^{*}+\delta t\right)}{a\left(t^{*}\right)}-1 \\
& <\frac{\left(\delta t \cdot l_{P}\right)}{\sqrt{3 / 8 \pi \Lambda}} \cdot \sqrt{\frac{1}{24 \pi a^{2}\left(t^{*}\right)}+\frac{1}{\Lambda} \cdot\left[\left(\rho_{r e l}\right)_{0}+\frac{a_{0}^{4}}{a^{6}\left(t^{*}\right)}+\left(\rho_{m}\right)_{o}-\frac{a_{0}^{3}}{a^{5}\left(t^{*}\right)}\right]} \\
& \underset{\delta t \rightarrow \varepsilon^{+}, \Lambda \neq \infty}{\longrightarrow} \frac{\left(\delta t \cdot l_{P} / a\left(t^{*}\right)\right)}{\sqrt{3 / 8 \pi \Lambda}} \cdot \sqrt{ } \cong \varepsilon^{+} \& \sqrt{ }=\sqrt{\frac{\left(\rho_{r e l}\right)_{0} a_{0}^{4}}{a^{4}\left(t^{*}\right)}+\frac{\left(\rho_{m}\right)_{0} a_{0}^{3}}{a^{3}\left(t^{*}\right)}}
\end{aligned}
$$

Here, we have that the coefficient, o, refers to the Planckian era, in general.

So in the initial phases of the big bang, with very large vacuum energy $\neq \infty$ and $0<a\left(t^{*}\right) \ll 1$, the following relation, which violates (signal) causality, is obtained for very small fluctuation $a\left(t^{*}\right)<l_{P}$ for $t^{*}<t_{P}$ = Planck time, and, which indicates that $[1]$

$$
\rho_{\text {rel }}=\left(\frac{a_{\text {present-era }}}{a(t)}\right)^{4} \cdot\left(\rho_{\text {rel }}\right)_{\text {present-era }}
$$

and

$$
\rho_{m}=\left(\frac{a_{\text {present-era }}}{a(t)}\right)^{3} \cdot\left(\rho_{m}\right)_{\text {present-era }}
$$

Using this above creates the following as plausible estimates which can be reviewed as needed. For large but not infinite temperatures, and for $\Lambda \approx c_{1} T^{\alpha}$

$$
\frac{\left(\delta t \cdot l_{P} / a\left(t^{*}\right)\right)}{\sqrt{3 / 8 \pi \Lambda}} \cdot \sqrt{\frac{\left(\rho_{r e l}\right)_{0} a_{0}^{4}}{a^{4}\left(t^{*}\right)}+\frac{\left(\rho_{m}\right)_{0} a_{0}^{3}}{a^{3}\left(t^{*}\right)}} \leq 10^{-4} \ll 1
$$

If we examine what happens with [4] a five dimensional version of the cosmological constant, we still get

$$
\frac{\left(\delta t \cdot l_{P} / a\left(t^{*}\right)\right)}{\sqrt{3 / 8 \pi\left|\Lambda_{5-\mathrm{dim}}\right|}} \cdot \sqrt{\frac{\left(\rho_{r e l}\right)_{0} a_{0}^{4}}{a^{4}\left(t^{*}\right)}+\frac{\left(\rho_{m}\right)_{0} a_{0}^{3}}{a^{3}\left(t^{*}\right)}}<10^{-4} \ll 1
$$

\section{Equation (7) to Equation (14) Are Connected with a Shift in the HUP as Delegated Below}

Start with

This leads to Planck's mass scaling, as alluded to by Barvinsky (2006) [5]

$$
\Lambda_{4-\text { dim }} \propto c_{2} T \underset{\text { Graviton-production }}{\longrightarrow} 360 m_{P}^{2} \ll c_{2} \cdot\left(T \sim 10^{32} \text { Kelvin }\right)
$$

We will be looking at the value of Equation (7), if $\phi \sim \xi^{+} \ll M_{\text {Planck }}$. In short, we have then that [6] [7] [8]

$$
\begin{aligned}
& (\Delta l)_{i j}=\frac{\delta g_{i j}}{g_{i j}} \cdot \frac{l}{2} \&(\Delta p)_{i j}=\Delta T_{i j} \cdot \delta t \cdot \Delta A \\
& \phi \sim \xi^{+} \ll M_{\text {Planck }}
\end{aligned}
$$


If we use the following, from the Roberson-Walker metric. [6] [7] [8]

$$
\begin{aligned}
& g_{t t}=1 \\
& g_{r r}=\frac{-a^{2}(t)}{1-k \cdot r^{2}} \\
& g_{\theta \theta}=-a^{2}(t) \cdot r^{2} \\
& g_{\phi \phi}=-a^{2}(t) \cdot \sin ^{2} \theta \cdot \mathrm{d} \phi^{2}
\end{aligned}
$$

Following Unruth [7] [8] write then, an uncertainty of metric tensor as, with the following inputs

$$
a^{2}(t)_{\min } \sim 10^{-110}, r \sim l_{P} \sim 10^{-35} \text { meters }
$$

Then, the surviving version of Equation (8) and Equation (9) is, then, if $\Delta T_{t t} \sim \Delta \rho$ [6] [7] [8]

$$
\begin{aligned}
& V^{(4)}=\delta t \cdot \Delta A \cdot r \\
& \delta g_{t t} \cdot \Delta T_{t t} \cdot \delta t \cdot \Delta A \cdot \frac{r}{2} \geq \frac{\hbar}{2} \\
& \Leftrightarrow \delta g_{t t} \cdot \Delta T_{t t} \geq \frac{\hbar}{V^{(4)}}
\end{aligned}
$$

This Equation (11) is such that we can extract, up to a point the HUP principle for uncertainty in time and energy, with one very large caveat added, namely if we use the fluid approximation of space-time [9] [10]

$$
T_{i i}=\operatorname{diag}(\rho,-p,-p,-p)
$$

Then by [9]

$$
\Delta T_{t t} \sim \Delta \rho \sim \frac{\Delta E}{V^{(3)}}
$$

Then,

$$
\begin{aligned}
& \delta t \Delta E \geq \frac{\hbar}{\delta g_{t t}} \neq \frac{\hbar}{2} \\
& \text { Unless } \delta g_{t t} \sim O(1)
\end{aligned}
$$

This behavior of the quantum uncertainty principle, as given will lead to the following inequalities.

\section{Equation (7) to Equation (14) Are Connected with a Shift in Delegated Inequalities Given Below}

This is assuming that one is having large, but non infinite temperatures which would not be in excess of, say $10^{\wedge} 34$ Kelvin.

We are also assuming a base line time unit of $t^{*}<t_{P} \sim 10^{-45} \mathrm{sec}$ and a time variance $\delta t \ll t^{*}<t_{P} \sim 10^{-45} \mathrm{sec}$.

So, the nomenclature of 0 put in denotes near Planckian physics conditions for a discrete equation model of Equation (2) leads to Dr. Dowker's paper on causal sets [2] which requires the following ordering with a relation $\prec$, where we assume that initial relic space time is replaced by an assembly of discrete 
elements, so as to create, initially, a partially ordered set $C$ :

1) If $x \prec y$ and $y \prec z$, then $x \prec z$;

2) If $x \prec y$ and $y \prec x$ then $x=y$ for $x$ and $y$ in a partially ordered set $C$;

3) For any pair of fixed elements $x$ and $z$ of elements in $C$, the set $\{y \mid x \prec y \prec z\}$ of elements lying in between $x$ and $z$ is finite, which is fulfilled by Equation (15) below.

$$
\frac{a\left(t^{*}+\delta t\right)}{a\left(t^{*}\right)}<1
$$

Items (1) and (2) about partially ordered sets above Equation (15) give us that we have $C$ as a partially ordered set and the third item permits local finiteness. This when combined with as a model for how the universe evolves via a scale factor equation permits violation of partial ordering. It is our contention that this will lead to the increase in entropy as the instanton is broken by Equation (15) above.

Another way to present this, and to get a tie into chaotic evolution is to make the following approximations to Equation (1) above: If $u=a^{-1}$

$$
\begin{aligned}
& \sqrt{\frac{\Lambda}{3}} \cdot \int \mathrm{d} t=-\int \frac{\mathrm{d} u}{\sqrt{\Theta}} \\
& \& \Theta=1+\frac{8 \pi}{\Lambda} \cdot\left(\left(\rho_{r e l}\right)_{0} a_{0}^{4} u^{4}+\left(\rho_{m}\right)_{0} a_{0}^{3} u^{3}\right)
\end{aligned}
$$

Integrating leads to the following polynomial expression for $u=a^{-1}$

$$
\begin{aligned}
& u^{9}+\frac{u^{8}}{a_{0}} \cdot A_{1}+\frac{u^{7}}{a_{0}^{2}} \cdot A_{2}-\frac{\Lambda}{8 \pi} \frac{u^{5}}{a_{0}^{4}} \cdot A_{3}-\frac{\Lambda}{8 \pi} \cdot \frac{u^{4}}{a_{0}^{5}} \cdot A_{4} \\
& +\left(\frac{\Lambda}{8 \pi}\right)^{2} \cdot \frac{u}{a_{0}^{8}} \cdot A_{5}+\left(\frac{\Lambda}{8 \pi}\right)^{2} \cdot \frac{t}{a_{0}^{9}} \cdot A_{6}=0
\end{aligned}
$$

We could go considerably higher in polynomial roots of Equation (16) above, depending upon the degree of accuracy we wished to obtain. This truncation so picked above is assuming a non infinite value of $u=a^{-1}$, as well as a non zero value, and non infinite value for the $\Lambda$ term. In doing so, we would obtain an extremely non standard evolution for the scale factor, assuming when we do so that

$$
\begin{aligned}
& A_{1}=\frac{9}{4} \cdot \frac{\left(\rho_{m}\right)_{0}}{\left(\rho_{r e l}\right)_{0}}, A_{2}=\left(\frac{\left(\rho_{m}\right)_{0}}{\left(\rho_{r e l}\right)_{0}}\right)^{2} \\
& A_{3}=\frac{1}{5} \cdot \frac{1}{\left(\rho_{r e l}\right)_{0}^{2}}, A_{4}=\frac{1}{4} \cdot \frac{\left(\rho_{m}\right)_{0}}{\left(\rho_{r e l}\right)_{0}^{2}} \\
& A_{5}=\frac{1}{\left(\rho_{r e l}\right)_{0}^{2}}, A_{5}=\frac{1}{\left(\rho_{r e l}\right)_{0}^{2}} \cdot \sqrt{\frac{\Lambda}{3}}
\end{aligned}
$$

Beginning with Mukhanov [11] setting his spatial dimension for a "particle" as $r \propto a \approx \varepsilon^{+}$, we look at how to implement Equation (15) above. We have that if we write 


$$
\begin{aligned}
& a\left(t^{*}+\delta t\right)<a\left(t^{*}\right) \\
& \Leftrightarrow \frac{1}{\Delta \widehat{\varepsilon}}<\frac{1}{\widehat{\varepsilon}_{1}} \\
& \Leftrightarrow \widehat{\varepsilon}_{1}<\Delta \widehat{\varepsilon}<\widehat{\varepsilon}_{2}
\end{aligned}
$$

The transition from $\hat{\varepsilon}_{1}=\hat{\varepsilon}_{2}-\Delta \hat{\varepsilon} \rightarrow \Delta \hat{\varepsilon}$ as $t^{*} \rightarrow t^{*}+\delta t$. This would correspond to the following picture. Have $\Delta \bar{\varepsilon}$ be the net energy density inside an instanton, with a boundary region of $\widehat{\varepsilon}_{2}-\Delta \widehat{\varepsilon} \geq 0$ energy density on the boundary of the Instanton. As the $\widehat{\varepsilon}_{2}-\Delta \widehat{\varepsilon} \rightarrow 0$, we have a release of $\Delta \widehat{\varepsilon}$ from the interior of the soliton (instanton) we can assume is in a Pre Planckian space-time. The following can be entertained, i.e. if we set energy density dimensions here as $\rho_{2}$ [12], we use the following form for information

$$
[\text { \# operations }]_{2} \approx \rho_{2} \cdot(c=1)^{2} t_{P}^{4} \leq 10^{120}
$$

This commences our treatment of the four dimensional discontinuity and the HUP, which we will then embed in a deterministic structure, in 5 dimensions [13]-[18].

\section{Setting the HUP, and Discontinuity in a 5-Dimensional Setting, as Part of Embedding to the Octonionic State}

This among other things is a fulfillment of the dream by Kaluza Klein [13] [14] [19], of sorts as far as how to unify Gravity and Electromagnetism in cosmology, but it has a much bigger cache than this, mainly as to understand the role of time, itself in quantum statistical ensembles, i.e. the idea of a deterministic large scale state, which would encompass quantum microstates in an ensemble within which the quantum microstates would be a way to analyze basic quantum thinking in terms of time dependence. In doing this, it also links itself to the question of why Schrodinger was so aghast at the idea of quantum jumping.

Let us now, briefly allude to the [13] [14] reference, namely:

Start with the idea of an embedding of four-dimensional space-time in a 5 dimensional time interval. [13] [14], and realize its inter connections with [15] [16], and [17], where $L=$ length of canonical metric in 5 Dimensional theory

$$
\begin{aligned}
& \mathrm{d} S_{5-\mathrm{dim}}^{2}=\frac{L^{2}}{l^{2}} \mathrm{~d} S_{4-\mathrm{dim}}^{2}-\left(\frac{L^{2}}{l^{2}}\right)^{2} \mathrm{~d} l^{2} \\
& x_{4}=l=h / m c \\
& \Lambda=3 / L^{2} \\
& L=\text { scale of universe potential well }
\end{aligned}
$$

And then we present, the five moment a as given by

$$
\begin{aligned}
& P^{\alpha}=\frac{2 L^{2}}{l^{2}} \cdot g^{\alpha \beta} \cdot \frac{\mathrm{d} x^{\beta}}{\mathrm{d} x} \\
& \& P_{l}=-\frac{2 L^{2}}{l^{4}} \cdot \frac{\mathrm{d} l}{\mathrm{~d} s}
\end{aligned}
$$

Then, if 


$$
\begin{aligned}
& P^{\alpha}=\frac{2 L^{2}}{l^{2}} \cdot g^{\alpha \beta} \cdot \frac{\mathrm{d} x^{\beta}}{\mathrm{d} x} \& P_{l}=-\frac{2 L^{2}}{l^{4}} \cdot \frac{\mathrm{d} l}{\mathrm{~d} s} \\
& \Rightarrow \int P_{A} \mathrm{~d} x^{A}=\int P_{\alpha} \mathrm{d} x^{\alpha}+\int P_{l} \mathrm{~d} l=0 \text { iff } \mathrm{d} S_{5-\text { dim }}^{2}=0 \\
& \Leftrightarrow l=l_{0} \exp ( \pm S / L) \&\left(\frac{\mathrm{d} l}{\mathrm{~d} S}\right)= \pm l / L
\end{aligned}
$$

One eventually, as given by [13] obtains the Heisenberg type of relations that

$$
\mathrm{d} p_{\alpha} \mathrm{d} x^{\alpha}=\hbar \cdot\left[\frac{n}{c} \cdot\left(\frac{\mathrm{d} l}{l}\right)^{2}\right]_{\alpha}
$$

In this case, looking at a rewrite of the time component of Equation (24) to read, approximately as [18]

$$
\mathrm{d} p_{0} \mathrm{~d} x^{0}=\hbar \cdot\left[\frac{n}{c} \cdot\left(\frac{\mathrm{d} l}{l}\right)^{2}\right]_{0}
$$

With the result that

$$
\begin{aligned}
& \mathrm{d} p_{\alpha} \mathrm{d} x^{\alpha}=\hbar \cdot\left[\frac{n}{c} \cdot\left(\frac{\mathrm{d} l}{l}\right)^{2}\right] \underset{\alpha \rightarrow 0}{\longrightarrow} \Delta E \Delta t \geq \frac{\hbar}{g_{t t}} \neq \hbar \\
& \text { Unless } g_{t t} \sim O(1)
\end{aligned}
$$

We urge the readers to consult [13] [14] [15] [16] [17], as well as [18] for what we are implying. Note also in [17] that Klein does a quantum mechanical treatment, which is in a sense has some elements of the later work on black body radiation problem.

i.e. what we have done is to say that Equation (26) establishes that the HUP is embedded within a deterministic structure in 5-dimensional Kaluza-Klein theory [13].

We argue that Equation (26) which is embedding the HUP, and in effect, time within a deterministic 5-dimensional structure, as given by [13] is in the end no different from the radical supposition given by Schrodinger as to quantum statistics, which was argued over in Solvay, as seen in [18] that the modeling of black body style quantum statistics, for a macro system was in a state which did not have an explicitly time dependent dynamic, i.e. as given in [19] by Shankar,

$$
\frac{\mathrm{d} E}{Q[\text { Thermal-cavity-Volume }]}=\rho(\omega) \mathrm{d} \omega=\frac{\hbar \omega \mathrm{d} \omega}{\pi^{2} c^{3} \cdot\left(\exp \left[\hbar \omega / k_{B} T\right]-1\right)}
$$

Having said this, and including in the description of embedding of the HUP in 4 space in quasi deterministic reasoning for 5-dimensions, we will the make the following assertions as to what this is saying about Octontonionic commutation relationships.

First, we will be examining what happens if

$$
\begin{aligned}
& \delta t \Delta E \geq\left.\frac{\hbar}{\delta g_{t t}}\right|_{\text {Pre-Octonionic }} \underset{\delta_{0} \text { phase-change }}{\longrightarrow} \delta t \Delta E \geq\left.\hbar\right|_{\text {Octonionic }} \\
& \text { with } \delta t \geq \frac{\hbar}{\delta g_{t t} \Delta E} \text { fixed }
\end{aligned}
$$


First a pre-quantum space time state, in which, in commutation relations [20] (Crowell, 2005) in the pre Octionic space time regime no approach to QM commutations is possible as seen by.

$$
\left[x_{j}, p_{k}\right] \neq-\beta \cdot\left(l_{\text {Planck }} / l\right) \cdot \hbar \cdot T_{i j k} \text { and does not } \rightarrow i \hbar \delta_{i, j}
$$

In the situation when we approach quantum "octonionic gravity applicable" geometry, Equation (29) becomes

$$
\left[x_{i}, p_{j}\right]=-\beta \cdot\left(l_{\text {Planck }} / l\right) \cdot \hbar \cdot T_{i j k} \underset{\text { approaching-flat-space-after- } \delta_{0}}{\longrightarrow} i \cdot \hbar \cdot \delta_{i, j}
$$

This commutation behavior should not be seen to contravene the structures given in [21] [22].

Equation (30) is such that even if one is in flat Euclidian space, and $i=j$, then if the phase transition from Pre Octonionic to Octonionic has occurred, and Equation (30) is key to what we say next here. i.e.

The point is this, i.e. by [19]

$$
\begin{aligned}
& \frac{\bar{p}_{j}}{m_{j}}=\frac{\hbar}{i \cdot m_{j}} \int \psi^{*} \frac{\partial \psi}{\partial x_{j}} \mathrm{~d} v \\
& =\frac{i}{\hbar} \int \psi^{*}\left(H_{\text {hamiltonian }} x_{j}-x_{j} H_{\text {hamiltonian }}\right) \psi \mathrm{d} v \\
& \frac{\mathrm{d} \bar{x}_{j}}{\mathrm{~d} t} \Rightarrow \bar{p}_{j}=m_{j} \frac{\mathrm{d} \bar{x}_{j}}{\mathrm{~d} t}
\end{aligned}
$$

Put this set of values of average momentum

$$
\begin{aligned}
& \bar{p}_{j}=m_{j} \frac{\mathrm{d} \bar{x}_{j}}{\mathrm{~d} t} \\
& \&\left[x_{i}, p_{j}\right]=-\beta \cdot\left(l_{\text {Planck }} / l\right) \cdot \hbar \cdot T_{i j k} \\
& \Rightarrow\left[x_{i}, \frac{\mathrm{d} \bar{x}_{j}}{\mathrm{~d} t}\right]=-\frac{\beta \cdot\left(l_{\text {Planck }} / l\right) \cdot \hbar \cdot T_{i j k}}{m_{j}}
\end{aligned}
$$

The last line of Equation (32) will be crucial to what we say next

$$
\begin{aligned}
& \frac{\mathrm{d} \bar{x}_{j}}{\mathrm{~d} t}=\sqrt{\frac{H_{\text {hamiltonian }}}{\sqrt{2 m_{j}}}} \approx \sqrt{\frac{\Delta E}{\sqrt{2 m_{j}}}} \\
& \Rightarrow\left[x_{i}, \sqrt{\frac{\Delta E}{\sqrt{2 m_{j}}}}\right]=-\frac{\beta \cdot\left(l_{\text {Planck }} / l\right) \cdot \hbar \cdot T_{i j k}}{m_{j}} \\
& \& \Delta E \approx \frac{\hbar}{\delta t \cdot \delta g_{t t}} \\
& \Rightarrow\left[x_{i}, \sqrt{\frac{\hbar}{\delta t \cdot \delta g_{t t} \cdot \sqrt{2 m_{j}}}}\right]=-\frac{\beta \cdot\left(l_{\text {Planck }} / l\right) \cdot \hbar \cdot T_{i j k}}{m_{j}}
\end{aligned}
$$

In doing this, we will make the final, identification

i.e.

$$
a\left(t^{*}+\delta \cdot t\right)<a\left(t^{*}\right) \Leftrightarrow \hat{\varepsilon}_{1}<\Delta \hat{\varepsilon}<\hat{\varepsilon}_{2}
$$

i.e. satisfying Equation (33) will enable to help us to consider the causal bar- 
rier situation as given in Equation (34) and this is the linkage between the idea of a causal discontinuity, Octonionic geometry and non commutativity, and the onset of new physics.

We claim that the details of reconciling Equation (33) and Equation (34) and the problem of finding a suitable time step, $\delta t$ which will be solidly linked to the embedding of the HUP, as is given in Equation (24) of our manuscript.

\section{Conclusion. What Equation (33) and Equation (34) Say about New Physics}

What we are being told, as to the relative position of the co-ordinates as to Pre-Planckian, to Planckian physics, at a causal barrier situation, just before the expansion of the Universe. i.e. decoding the information in Equation (33) and Equation (34) will be crucial to all that.

The main point in decoding Equation (33) and Equation (34) will be in the assembling of a suitable value for the minimum time step, i.e. what we suspect is that an optimal $\delta t$ will be heavily dependent upon making sense of both Equation (33) and Equation (34) with $\delta t$, a bi-product of the embedding procedures given in Equation (24) and Equation (25) starting with the procedures given in Equation (21) above.

Secondly, if we wish to go to the idea of many mini black holes, initially, say of mass one Plank mass each, initially, we can look at

$$
\begin{aligned}
& S \leq 2 \pi R E /(\hbar \cdot c) \\
& R \approx l_{\text {Planck }} \& E \propto \text { mass } \cdot c^{2} \\
& \text { mass } \propto M_{\text {Planck }} \approx \# \cdot m_{\text {graviton }} \approx 2.17645 \times 10^{-5} \text { grams } \\
& m_{\text {graviton }} \approx 10^{-65} \text { grams } \\
& \& 10^{20} \leq \# \leq 10^{60}
\end{aligned}
$$

Equation (35) is relevant right after a presumed shift after a causal discontinuity. And it would be

$$
\begin{aligned}
& S \leq 2 \pi R E /(\hbar \cdot c) \\
& R \approx l_{\text {Planck }} \\
& \delta t \Delta E \geq\left.\frac{\hbar}{\delta g_{t t}}\right|_{\text {Pre-Octonionic }} \underset{\delta_{0} \text { phase-change }}{\longrightarrow} \delta t \Delta E \geq\left.\hbar\right|_{\text {Octonionic }} \\
& \Delta E \geq \frac{\hbar}{\delta t \delta g_{t t}} \\
& \Delta S \approx 2 \pi R E /(\hbar \cdot c) \approx 2 \pi l_{\text {Planck }} /\left(\delta t \cdot \delta g_{t t} \cdot c\right)
\end{aligned}
$$

This above should be compared to the following, from Octonionic reasoning

$$
\begin{aligned}
& {\left[x_{i}, \sqrt{\frac{\Delta E}{\sqrt{2 m_{j}}}}\right]=-\frac{\beta \cdot\left(l_{\text {Planck }} / l\right) \cdot \hbar \cdot T_{i j k}}{m_{j}} \& \Delta E \approx \frac{\hbar}{\delta t \cdot \delta g_{t t}}} \\
& \Rightarrow\left[x_{i}, \sqrt{\frac{\hbar}{\delta t \cdot \delta g_{t t} \cdot \sqrt{2 m_{j}}}}\right]=-\frac{\beta \cdot\left(l_{\text {Planck }} / l\right) \cdot \hbar \cdot T_{i j k}}{m_{j}}
\end{aligned}
$$


The key point is that the presumed massive increase in entropy, due to $\delta t$ say at the boundary of a Causal structure would be also reflected in the aftermath of making sense of inputs of $\delta t$ in a Pre-Octonionic to Octonionic geometrical setting. We state that the final details would also have $\delta t$ heavily influenced by the results of Equation (24) from Wesson, which we put in, again, namely due to details give in [13] and this is the first part of Equation (24)

$$
\mathrm{d} p_{\alpha} \mathrm{d} x^{\alpha}=\hbar \cdot\left[\frac{n}{c} \cdot\left(\frac{\mathrm{d} l}{l}\right)^{2}\right]_{\alpha}
$$

The point is that this 5-dimensional equation, a deterministic structure from Kaluza Klein theory, in its $\alpha=0$ setting, is crucial for the formation of $\delta t$ And, that $\delta t$ presumably helps in the formation of emergent gravity, along the lines also implied by [14] [15] [16] [17] [18].

We also point out the importance in all of these in keeping fidelity with [23] as what we are referencing is very close in subject to the possibilities so outlined.

References [24] [25], and [26] outline key experimental gravity considerations which we have to keep in mind when reviewing the suppositions so outlined. [14] and [26] are LIGO, and [25] is a very well thought out set of testing protocol we need to keep in mind as brought up by Corda.

The reference [27], by Kieffer and Starobinsky is a partial overlap with our ideas, although with a substantially different flavor.

References [28] [29] [30], and [31] are foundational considerations as to the main stay of quantum weirdness we need not to forget.

\section{Acknowledgements}

This work is supported in part by National Nature Science Foundation of China grant No. 11375279.

\section{Conflicts of Interest}

The authors declare no conflicts of interest regarding the publication of this paper.

\section{References}

[1] Frampton, P. and Baum, L. (2007) Turnaround in Cyclic Cosmology. Physical Review Letters, 98, Article ID: 071301.

[2] Dowker, H.F. (2005) Causal Sets and the Deep Structure of Spacetime. In: Ashtekar, A., Ed., 100 Years of Relativity Space-Time Structure: Einstein and Beyond, World Press Scientific, Singapore. https://doi.org/10.1142/9789812700988_0016

[3] Park, D.K., Kim, H. and Tamarayan, S. (2002) Nonvanishing Cosmological Constant of Flat Universe in Brane World Scenarios. Physics Letters B, 535, 5-10. https://doi.org/10.1016/S0370-2693(02)01729-X

[4] Beckwith, A.W. (2008) Symmetries in Evolving Space-Time and Their Connection to High-Frequency Gravity Wave Production. AIP Conference Proceedings, 969, 1018-1026. https://doi.org/10.1063/1.2844938 
[5] Barvinsky, A., Kamenschick, A. and Yu, A. (2006) Thermodynamics from Nothing: Limiting the Cosmological Constant Landscape. Physical Review D, 74, Article ID: 121502, Rapid Communications. https://doi.org/10.1103/PhysRevD.74.121502

[6] Beckwith, A. (2016) Gedanken Experiment for Refining the Unruh Metric Tensor Uncertainty Principle via Schwartz Shield Geometry and Planckian Space-Time with Initial Nonzero Entropy and Applying the Riemannian-Penrose Inequality and Initial Kinetic Energy for a Lower Bound to Graviton Mass (Massive Gravity). Journal of High Energy Physics, Gravitation and Cosmology, 2, 106-124. https://doi.org/10.4236/jhepgc.2016.21012

[7] Unruh, W.G. (1986) Why Study Quantum Theory? Canadian Journal of Physics, 64, 128-130. https://doi.org/10.1139/p86-019

[8] Unruh, W.G. (1986) Erratum: Why Study Quantum Gravity? Canadian Journal of Physics, 64, 128. https://doi.org/10.1139/p86-019

[9] Kolb, E., Pi, S. and Raby, S. (1984) Phase Transitions in Super Symmetric Grand Unified Models. In: Fang, L. and Ruffini, R., Eds., Cosmology in the Early Universe, World Press Scientific, Pte. Ltd. Co., Singapore, 45-70.

[10] Giovannini, M. (2008) A Primer on the Physics of the Cosmic Microwave Background. World Press Scientific, Hackensack, New Jersey. https://doi.org/10.1142/6730

[11] Viatcheslav, M. (2005) Physical Foundations of Cosmology. Cambridge University Press, Cambridge. https://doi.org/10.1017/CBO9780511790553

[12] Lloyd, S. (2002) Computational, Capacity of the Universe. Physical Review Letters, 88, Article ID: 237901. https://arxiv.org/abs/quant-ph/0110141 https://doi.org/10.1103/PhysRevLett.88.237901

[13] Wesson, P. (2006) 5-Dimensional Physics: Classical and Quantum Consequences of Kaluza Klein Cosmology. World Scientific Publishing Company, Singapore.

[14] Kaluza, T. (1921) Zum Unitätsproblem in der Physik. Sitzungsber. Preuss. Akad. Wiss., Berlin, 966-972. https://archive.org/details/sitzungsberichte1921preussi

[15] Klein, O. (1926) Quantentheorie und fünfdimensionale Relativitätstheorie. Zeitschrift für Physik A, 37, 895-906. https://doi.org/10.1007/BF01397481

[16] Wesson, P.S. (1999) Space-Time-Matter, Modern Kaluza-Klein Theory. World Scientific, Singapore. https://doi.org/10.1142/3889

[17] Klein, O. \{1931) Zur quantenmechanischen Begründung des zweiten Hauptsatzes der Wärmelehre. Zeitschrift für Physik, 72, 767-775.

[18] Beckwith, A.W. (1927) History Lessons from the 5th Solvay Meeting 1927. http://vixra.org/abs/1708.0399

[19] Shankar, R. (1994) Principles of Quantum Mechanics. 2nd Edition, Springer Verlag, Heidelberg. https://doi.org/10.1007/978-1-4757-0576-8

[20] Crowell, L. (2005) Quantum Fluctuations of Spacetime. World Scientific Series in Contemporary Chemical Physics, Volume 25, World Scientific Publishing Company, Singapore.

[21] Wilson, R. (2008) Octonions. http://www.maths.qmul.ac.uk/ raw/talks_files/octonions.pdf

[22] Dixon, G.M. (1994) Division Algebras: Octonions Quaternions Complex Numbers and the Algebraic Design of Physics. Kluwer Academic Publishers, London. https://doi.org/10.1007/978-1-4757-2315-1

[23] Clarkson, C. and Seahra, S. (2007) A Gravitational Wave Window on Extra Dimensions. Classical and Quantum Gravity, 24, F33-F40. 
https://doi.org/10.1088/0264-9381/24/9/F01

[24] Abbott, B.P., et al. (2016) Observation of Gravitational Waves from a Binary Black Hole Merger. Physical Review Letters, 116, Article ID: 061102.

https://physics.aps.org/featured-article-pdf/10.1103/PhysRevLett.116.061102

[25] Corda, C. (2009) Interferometric Detection of Gravitational Waves: The Definitive Test for General Relativity. International Journal of Modern Physics D, 18, 2275-2282. https://doi.org/10.1142/S0218271809015904

[26] Abbot, B.P., et al. (2016) GW151226: Observation of Gravitational Waves from a 22-Solar-Mass Binary Black Hole Coalescence. Physical Review Letters, 116, Article ID: 241103. https://doi.org/10.1103/PhysRevLett.116.241103

[27] Kiefer, C., Polarski, D. and Starobinsky, A.A. (2000) Entropy of Gravitons Produced in the Early Universe. Physical Review D, 62, Article ID: 043518.

[28] t'Hooft, G. (1999) Quantum Mechanics as a Dissipative Deterministic System. http://arxiv.org/PS_cache/gr-qc/pdf/9903/9903084v3.pdf

[29] Schrodinger, E. (1952) Are There Quantum Jumps. The British Journal for the Philosophy of Science, 4, 109-123, 233-247.

[30] Malomed, B. (2005) Nonlinear Schrödinger Equations. In: Scott, A., Ed., Encyclopedia of Nonlinear Science, Routledge, New York, 639-643.

[31] Pashby, T. (2005) Time and the Foundations of Quantum Mechanics. http://philsci-archive.pitt.edu/10666/1/Pashby_2014_Time_Foundations_QM.pdf 